\title{
Comparative Evaluation of a High Operating Temperature Midwave Infrared Detector for Automated Non-Destructive Inspection of Composite Damage
}

\author{
Jaslyn Graya ${ }^{*}$, Michael Woodrow ${ }^{b}$, Cédric Rosaliec ${ }^{c}$ Nik Rajic ${ }^{d}$ \\ Defence Science and Technology Group, 506 Lorimer Street, Fishermans Bend, VIC, 3207, \\ Australia \\ ajaslyn.gray@dst.defence.gov.au,bmichael.woodrow@dst.defence.gov.au, \\ ccedric.rosalie@dst.defence.gov.au, dnik.rajic@dst.defence.gov.au
}

(C) 2020 Commonwealth of Australia

Keywords: Line Scan Thermography, Composites, Dynamic Pulse Phase Thermography, Non-destructive Testing, Barely Visible Impact Damage, Robotic Inspection

\begin{abstract}
A new high operating temperature (HOT) midwave infrared (MWIR) imaging core is experimentally evaluated for use in automated inspection of composite impact damage by line scan thermography (LST). This evaluation is undertaken as part of a broader effort to develop an autonomous inspection capability for aerospace composite structures, deployable by ground and aerial robotic systems. The performance of the HOT MWIR core is assessed against a highperformance cooled photon-detector camera, an uncooled microbolometer core and an uncooled microbolometer camera, on two carbon epoxy laminate test specimens: one containing flatbottom-hole synthetic defects and the other barely visible impact damage (BVID) introduced by controlled low-velocity impact. These test panels are scanned using a 3-axis robotic LST apparatus, at speeds of 25 and $100 \mathrm{~mm} / \mathrm{s}$. The HOT MWIR core is shown to match the detection performance of the cooled camera, and to significantly outperform both microbolometers. The high performance of this core combined with its relatively low mass, size and power consumption offers an encouraging basis for the development of a drone-deployable LST inspection capability.
\end{abstract}

\section{Introduction}

The use of carbon fibre reinforced polymer (CFRP) components in aircraft construction has increased steadily over time. For example, a typical airframe manufactured in the 1970s contained approximately 5\% by weight in CFRP, whilst a modern A350 contains over 50\% [1]. This trend is continuing. CFRPs have many practical advantages over metals for aircraft construction, including a higher strength and stiffness to weight ratio and better corrosion and fatigue resistance. However, they are more prone to damage by impact. Barely visible impact damage (BVID) [2] is of particular concern as this type of damage is difficult to detect by visual inspection and if left undetected can result in component failure. This has driven interest in more rapid and reliable methods of BVID detection, sizing and analysis. Automated and autonomous inspection by aerial and ground robotic systems $[3,4]$ is an emerging area of interest with the potential to significantly reduce inspection time and cost relative to current practice.

Several different non-destructive testing (NDT) methods have been applied to BVID inspection [5]. These can be broadly categorised into contact or non-contact methods; contact methods, e.g. ultrasonic, eddy current and liquid penetrant, are generally considered to be more 
accurate but time consuming, whereas non-contact methods are generally faster and more versatile [6]. Active infrared thermography (AIT) [7] is a key non-contact method. AIT uses an external energy source, most commonly a high intensity lamp, to heat a subject and the resulting surface temperature distribution is observed with an infrared camera. The delaminations associated with BVID impede through-thickness heat flow resulting in a locally lower rate of cooling, thus producing a surface temperature contrast.

Line Scan Thermography (LST) is a variant of AIT wherein a line source of heat moves at constant speed over the test area which is observed by an infrared camera moving in unison with the source [8]. LST has the capacity to inspect large areas of structure at relatively high speed and the potential to be deployed by either an unmanned aerial vehicle (UAV) or unmanned ground vehicle (UGV) [9]. Preliminary work toward realising such a capability was undertaken using a 3DOF Cartesian robot and was reported in [10]. In this previous work, experimental testing on laminate samples containing BVID produced generally encouraging results, but it also demonstrated that a relatively high-performance infrared imaging capability is necessary to achieve satisfactory detection results; specifically, a cooled photon imager was shown to significantly outperform a microbolometer. However, cooled detectors are relatively large and expensive and for these reasons impractical for the drone-based inspection application under consideration.

The recent emergence of high operating temperature (HOT) medium wave infrared (MWIR) imaging core technology offers a potential solution. These HOT MWIR cores employ a type II strained layer superlattice (T2SL) sensor material which suppresses dark current noise at significantly higher detector operating temperatures $(\sim 120 \mathrm{~K})$ than are required for traditional cooled photon detectors $(\sim 77 \mathrm{~K})[11]$. This reduced cooling requirement results in a device which is comparatively much smaller, lighter, and power efficient, whilst achieving similar imaging performance.

The principal aim of the present work is to experimentally evaluate the LST inspection performance of this new class of infrared imaging device against a conventional highperformance cooled photon camera and two uncooled microbolometers. Complementary multiphysics-based simulations of the LST process are also undertaken to verify a new image processing approach called dynamic pulse phase thermography (DPPT) which is used to extract defect signatures from raw data obtained from the different imaging devices.

\section{Experimental Testing}

LST experiments were undertaken using a 3-axis Cartesian robotic facility illustrated in Fig. $1(\mathrm{a})$.

Translation along the three axes is achieved using brushless DC motors which are driven through a programable logic controller (PLC). The LST sensing apparatus comprises an infrared imager and luminaire installed at the base of the z-axis robot arm. The luminaire (Fig. 1(b)) contains a polished metal parabolic reflector which focuses light from a $118 \mathrm{~mm}$ long $150 \mathrm{~W}$ quartz-halogen lamp positioned at the reflectors focal point, to a line width of $\sim 20 \mathrm{~mm}$ at a distance $500 \mathrm{~mm}$ from the lamp. Previous work has shown that this line width is sufficient to produce reliable indications of the composite damage targeted in the present investigation [10].

Table 1 lists the four IR imaging devices considered in this study along with relevant specifications. The last three rows of this table illustrate the profound difference in size, mass and power consumption of uncooled HOT MWIR detectors compared to a typical cooled camera. Henceforth, these imagers will be referred to by the designation given in Table 1. 
(a)

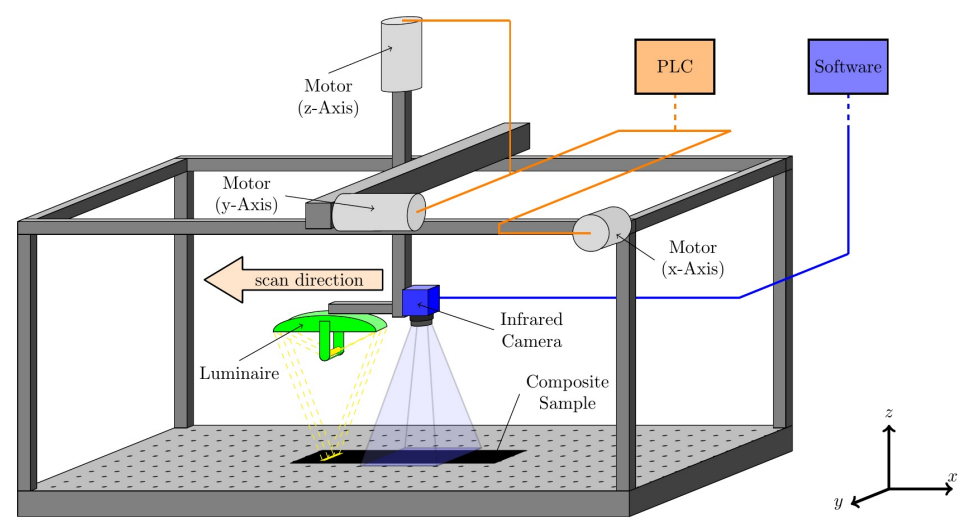

(b)

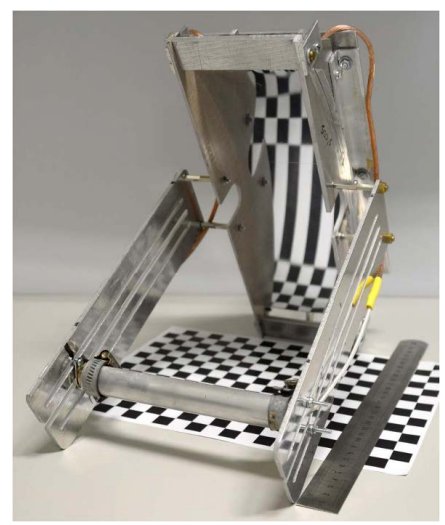

Fig. 1: (a) Schematic of 3-axis Cartesian robot showing key LST system components.

(b) Luminaire comprising a lamp and parabolic reflector, with $300 \mathrm{~mm}$ ruler shown for scale.

Table 1: Infrared imager specifications.

\begin{tabular}{c|cccc}
\hline Type & Microbolometer & Microbolometer & $\begin{array}{c}\text { Cooled } \\
\text { Photon detector }\end{array}$ & $\begin{array}{c}\text { HOT } \\
\text { Photon detector }\end{array}$ \\
\hline Manufacturer and model & FLIR Boson A640 & Xenics Gobi 640 & FLIR SC6000 & FLIR Neutrino LC \\
\hline Designation & $\mu$ B-VOx & $\mu B-a S i$ & PD-cooled & PD-HOT \\
\hline Detector & Vanadium Oxide & Amorphous Silicon & Indium Antimonide & HOT MWIR \\
\hline Spectral range $[\mu \mathrm{m}]$ & $7.5-13.5$ & $8.0-14.0$ & $3.0-5.0$ & $3.4-5.0$ \\
\hline Published NETD $[\mathrm{mK}]$ & 50 & 55 & 18 & 30 \\
\hline Array size & $640 \times 512$ & $640 \times 480$ & $640 \times 512$ & $640 \times 512$ \\
\hline Frame rate $[\mathrm{Hz}]$ & 60 & 50 & 100 & 60 \\
\hline Dimensions $((\mathrm{L} \times \mathrm{W} \times \mathrm{H}))[\mathrm{mm}]$ & $21 \times 21 \times 11$ & $79 \times 49 \times 49$ & $320 \times 141 \times 159$ & $79 \times 45.5 \times 61$ \\
\hline Mass $[\mathrm{g}]$ & $7.5($ without lens) & 263 & 4500 & 380 \\
\hline Power $[\mathrm{W}]$ & +0.5 & 4.5 & 50 & 4.5
\end{tabular}

\section{Composite test samples}

Two composite panels were examined in this study (see Fig. 2). The first panel is a rectangular laminate $330 \times 240 \times 2.5 \mathrm{~mm}$ in size and consisting of 9 plies of M18/1/43\% G939 carbon-epoxy biaxial pre-preg in the layup $[45 / 0 / 0 /-45 / 0 /-45 / 0 / 0 / 45]$. This panel contains six flat bottom holes $(\mathrm{FBH})$ of 5, 10, and $20 \mathrm{~mm}$ diameters at two different depths corresponding to a residual panel thickness of 1 and $1.5 \mathrm{~mm}$, as illustrated in Fig. 2(a). The second panel is also a rectangular, $470 \times 100 \times 4 \mathrm{~mm}$ in size and consists of 30 plies of IM7/977-3 carbon-epoxy prepreg in the lay-up $[45 / 0 / 0 /-45 / 90]_{\mathrm{s} 3}$. This second laminate contains a single BVID feature introduced by a $10 \mathrm{~J}$ controlled low-velocity impact. The presence of BVID in this panel was previously verified by flash thermography and pulse-echo ultrasonic testing, with indications produced by these methods shown in Fig. 2(b). The inspection surfaces of both samples were given a thin coating of high-emissivity matt black paint in preparation for LST testing. 
(a)

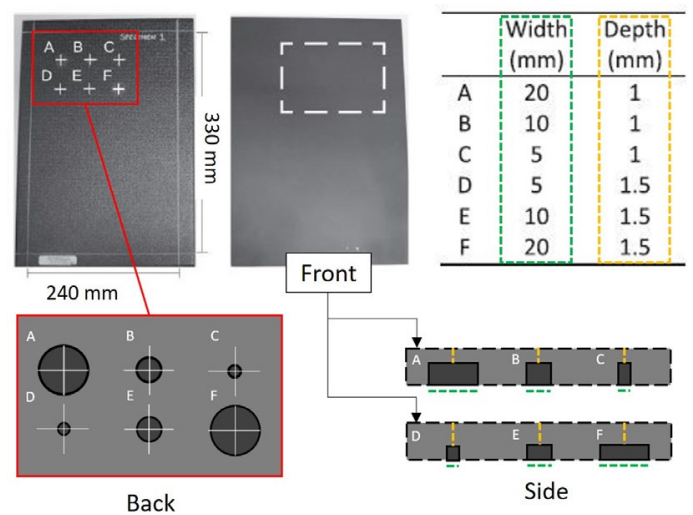

(b)

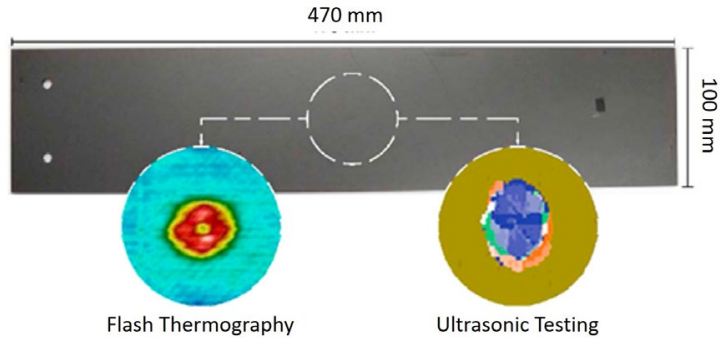

Fig. 2: (a) Composite panel containing six FBH defects. Back face showing FBH and front face with high emissivity paint coating. White dotted rectangle indicates area where defects are located. (b) BVID panel with damage confirmed by flash thermography and ultrasonic testing.

\section{Test and Processing Methodology}

LST inspections of the FBH and BVID test panels were undertaken using the four previously described imagers. A single scan consisted of one sweep of the sensor head in the x-direction at a constant speed of $25 \mathrm{~mm} / \mathrm{s}$ and $100 \mathrm{~mm} / \mathrm{s}$. Data acquired from each scan was post-processed using DPPT to produce a set of phase maps that were used as the basis for the performance comparison between detectors. The DPPT approach was detailed previously in [10]. For the purposes of the present article the salient feature of DPPT is that it maps thermal contrasts produced by sub-surface defects into dipole-like phase signatures, as will become clear in the following sections.

\section{Numerical Simulations}

Numerical simulations of the heat diffusion physics associated with an LST inspection were undertaken for two reasons: (i) to produce synthetic data for verification that the DPPT algorithm is functioning correctly and (ii) to foster insight into the LST process and in particular the effect of scan speed on the defect signature. The simulations were conducted using the COMSOL 5.3 software package. Only the FBH sample was considered and was modelled as a threedimensional transversely isotropic plate, with Table 2 listing the relevant material properties used for the simulation. The heat source was modelled as a Gaussian pulse (see Fig. 3) with a full-width at half-maximum dimension of $20 \mathrm{~mm}$. The laminate was meshed using tetrahedral elements and the solution was advanced in time steps of $0.01 \mathrm{~s}$. 


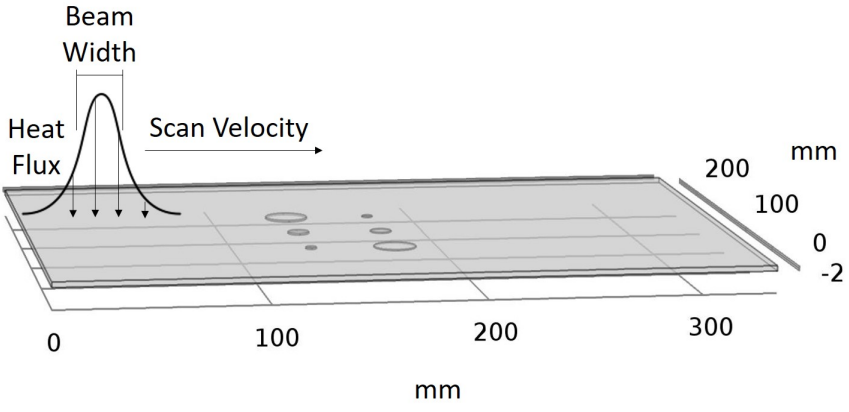

Fig. 3: Modelled plate geometry with key parameters.
Table 2: Material properties and simulation parameters.

\begin{tabular}{l|l}
\hline \multicolumn{2}{l}{ Material properties and parameters } \\
\hline Density $(\boldsymbol{\rho})\left[\mathrm{kg} / \mathrm{m}^{3}\right]$ & 1530 \\
\hline Heat capacity $(\mathrm{Cp})[\mathrm{J} /(\mathrm{kg} \mathrm{K})]$ & 950 \\
\hline $\begin{array}{l}\text { Lateral thermal conductivity } \\
(\mathrm{Kx}, \mathrm{Ky})[\mathrm{W} / \mathrm{mK}]\end{array}$ & 3.19 \\
\hline $\begin{array}{l}\text { Thickness thermal conductivity } \\
(\mathrm{Kz})[\mathrm{W} / \mathrm{mK}]\end{array}$ & 0.57 \\
\hline Scan velocity $[\mathrm{mm} / \mathrm{s}]$ & 25,100 \\
\hline Beam width $[\mathrm{mm}]$ & 20 \\
\hline Defect diameter $[\mathrm{mm}]$ & $20,10,5$ \\
\hline
\end{tabular}

Two scan velocities were considered in the simulation: $25 \mathrm{~mm} / \mathrm{s}$ and $100 \mathrm{~mm} / \mathrm{s}$, which correspond to the velocities also considered in the experimental work. Fig. 4(a) shows a representative surface temperature distribution from a simulation conducted at a $25 \mathrm{~mm} / \mathrm{s}$ scan speed, at a time $\sim 8$ seconds after the source has passed over the centre of the FBH region, which corresponds to peak contrast for the FBH signatures. A $1.35 \mathrm{~s}$ time window corresponding to when the defects are in the field of view of the IR imager during an experimental scan (between 14.65-16 s on the scale shown in Fig. 4(a)) was processed using DPPT to produce the phase map in Fig. 4(b).

(a)

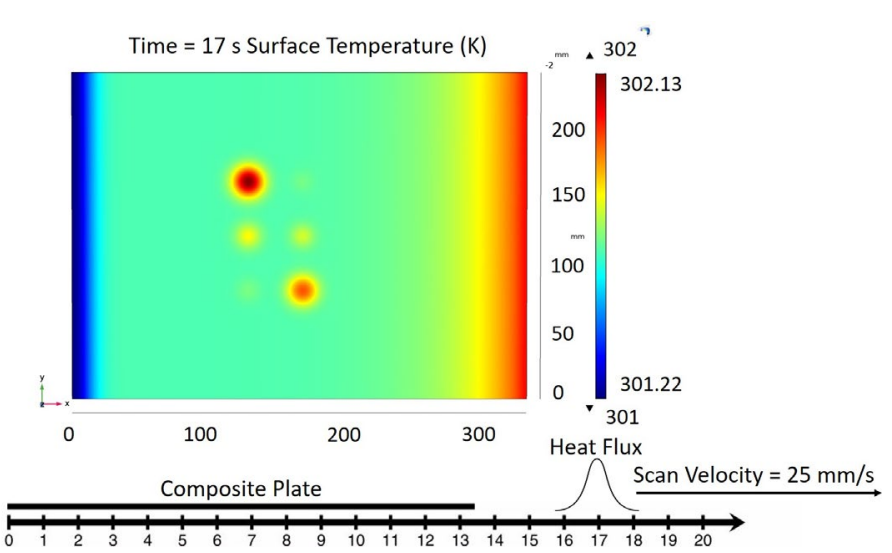

(b)

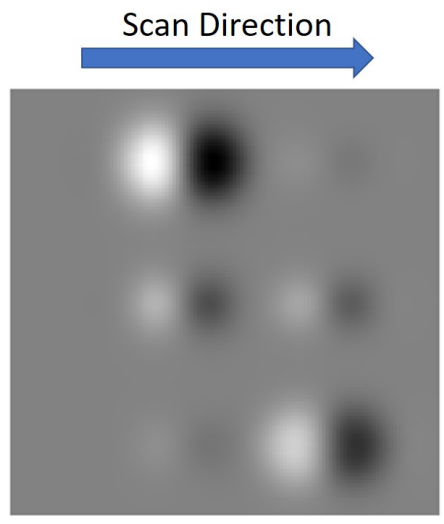

Fig. 4: (a) Surface temperature distribution obtained from simulation of a $25 \mathrm{~mm} / \mathrm{s}$ scan of FBH panel; timeline indicates heat flux position relative to panel.

(b) Corresponding DPPT phase map at $945 \mathrm{mHz}, 120 \mathrm{~mm}$ square region of interest.

The circular dipole signatures in Fig. 4(b) closely resemble experimentally obtained signatures reported in [10] and thus confirm proper functioning of the DPPT algorithm. Additionally, this result provides validation of the modelling capability, which is important for future development of this approach in allowing different inspection parameters and scenarios to be investigated in a simulated environment with confidence. 


\section{Results and Discussion}

Experimentally obtained FBH panel scan results corresponding to the four different IR imagers are compared in Fig. 5. These scans were undertaken at 25 and $100 \mathrm{~mm} / \mathrm{s}$, and the results correspond to the phase at $945 \mathrm{mHz}$, obtained from DPPT applied to a $1.35 \mathrm{~s}$ data window.

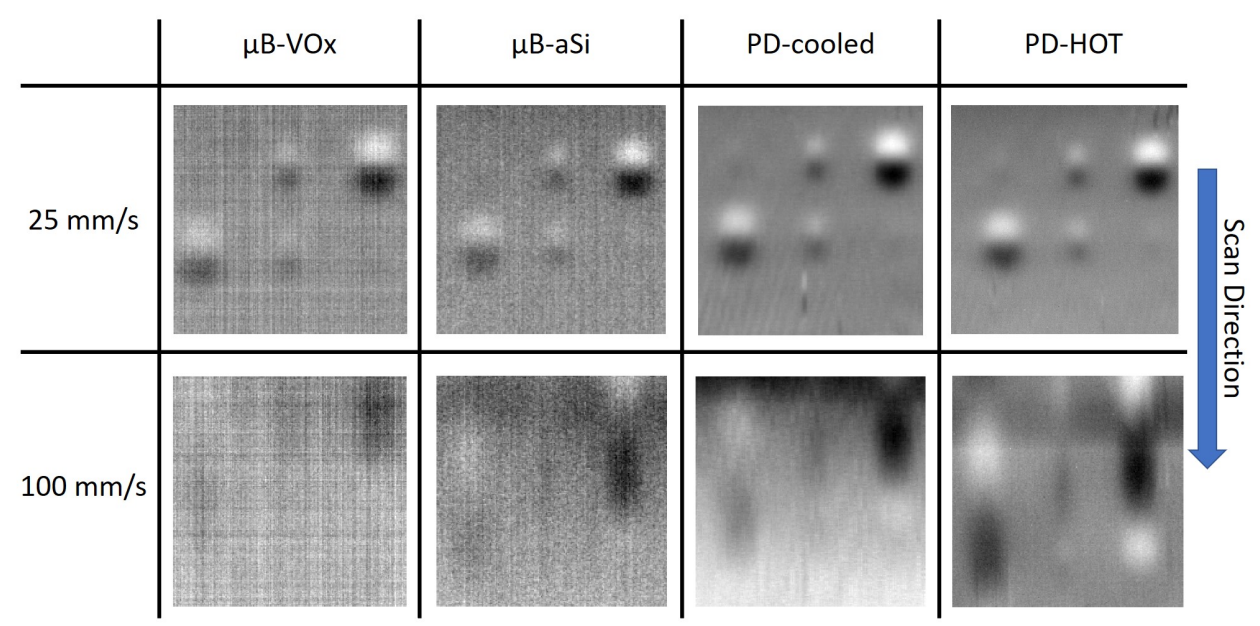

Fig. 5: Phase maps corresponding to FBH panel scanned at $25 \mathrm{~mm} / \mathrm{s}$ and $100 \mathrm{~mm} / \mathrm{s}$ for each IR imager. The region of interest is square with side dimension of $120 \mathrm{~mm}$.

These results show that the $20 \mathrm{~mm}$ and $10 \mathrm{~mm}$ diameter $\mathrm{FBH}$ defects are well resolved at the slowest scan speed for all imagers. However, for the $5 \mathrm{~mm}$ diameter defects, signatures are discernible, albeit faintly, only for the PD-cooled and PD-HOT imager results. The performance gap between these devices and the two microbolometers is more significant in the high scanspeed results (bottom row). Here, even the larger diameter defects are barely visible in the $\mu \mathrm{B}-$ VOx results, and only marginally more visible in the $\mu \mathrm{B}$-aSi results. The PD-HOT imager yields noticeably better results than the PD-cooled camera, which is interesting given the relative noise equivalent temperature difference (NETD) specifications of these devices (see Table 1). It is to be noted that the failure to resolve between the two rows of defects at the $100 \mathrm{~mm} / \mathrm{s}$ scan speed is due to a trade-off between scan speed and spatial resolution in LST, an issue that will be considered more thoroughly in a separate study.

The experimental results obtained from the PD-HOT core and PD-cooled camera for the FBH panel were also compared to corresponding predictions from the previously described numerical model, as shown in Fig. 6. This comparison illustrates remarkably good correlation, which provides further confidence in the developed model.

Fig. 7 shows results obtained for the BVID panel using the PD-HOT and PD-cooled imagers, at scan speeds of 25 and $100 \mathrm{~mm} / \mathrm{s}$. Signatures corresponding to the BVID are discernible in all four cases, but are strongest at the faster scan speed, presumably because of the shorter time available for heat diffusion. This result is encouraging from the viewpoint of developing a rapid inspection capability deployable by drone, for which HOT-MWIR imaging core technology appears well suited. 


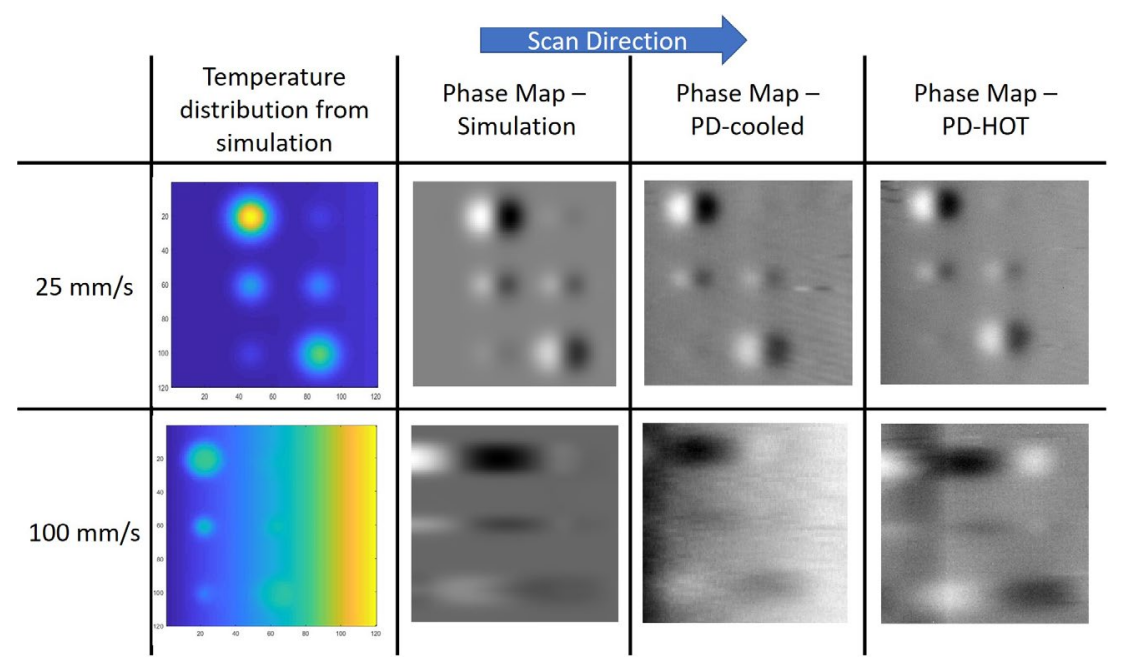

Fig. 6: Comparison of phase maps obtained from simulation and experimentally for the $P D$ cooled and PD-HOT imagers at scan speeds of $25 \mathrm{~mm} / \mathrm{s}$ and $100 \mathrm{~mm} / \mathrm{s}$. The region of interest and time window is unchanged from the previously described case.

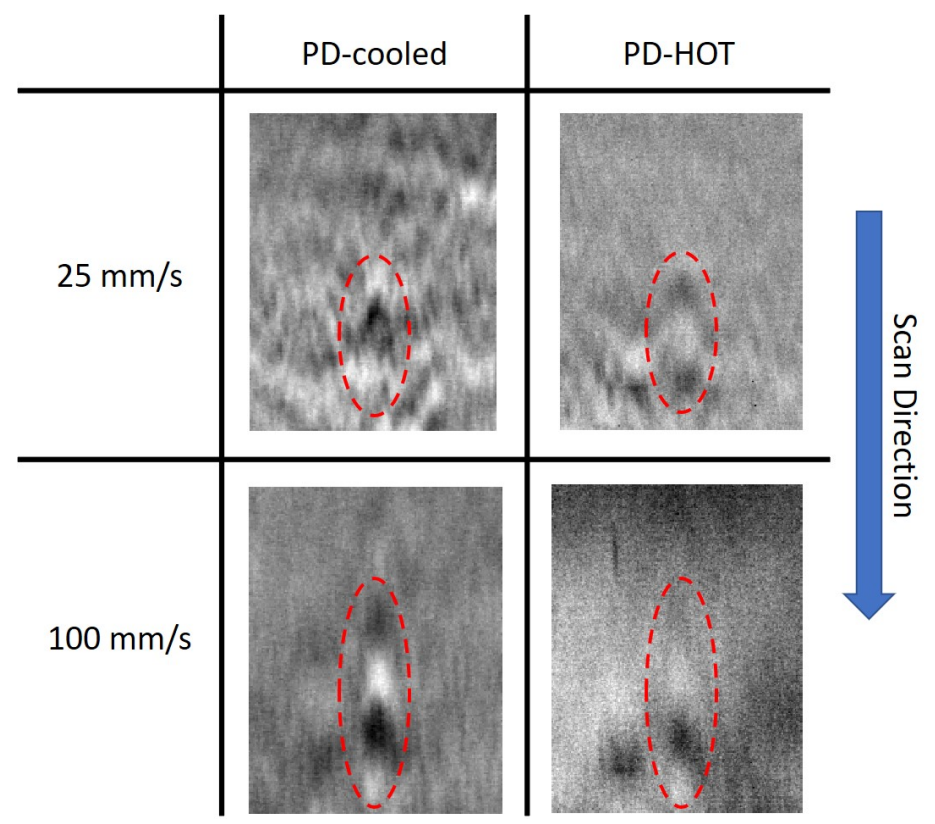

Fig. 7: Phase maps corresponding to BVID panel scanned at scan speeds of $25 \mathrm{~mm} / \mathrm{s}$ and 100 $\mathrm{mm} / \mathrm{s}$ using the PD-cooled and PD-HOT imagers. Dashed ellipse outlines defect signature ellipse. The region of interest is $85 \mathrm{~mm} \times 110 \mathrm{~mm}$ and observation time was 1.35 seconds.

\section{Conclusion}

A new high operating temperature mid-wave infrared imaging core has been experimentally evaluated for use in rapid automated inspection of composite damage by line scan thermography. The performance of this device was tested against an amorphous silicon microbolometer, a vanadium oxide microbolometer and a mid-wave cryogenically-cooled photon detector camera on composite laminates with flat-bottom-hole synthetic defects and barely visible impact damage. On both laminates the imaging core significantly outperformed the microbolometers and yielded comparable performance to the cooled camera. Given this core is a fraction of the 
mass and size of a typical cooled camera and consumes considerably less power, these results are encouraging for the development of a drone-deployable composite inspection capability using line scan thermography.

\section{Acknowledgment}

The authors acknowledge Mr. Chris Brooks, Mr. Daniel Bitton and Mr. Stephen van der Velden for their assistance in the experimental work.

\section{References}

[1] Mouritz, Adrian P, Introduction to aerospace materials, Elsevier, 2012. https://doi.org/10.1533/9780857095152

[2] Morton J, Godwin EW, Impact response of tough carbon fibre composites, Composite Structures, 1989 Jan 1;13(1):1-9. https://doi.org/10.1016/0263-8223(89)90069-X

[3] Mavromatidis, L.E. \&Dauvergne, J.-L \&Lunazzi, Renato \&Batsale, Jean-Christophe, First experiments for the diagnosis and thermophysical sampling using pulsed IR thermography from unmanned aerial vehicle (UAV), Quantitative InfraRed Thermography, 2014.

https://doi.org/10.21611/qirt.2014.213

[4] V. Kostopoulos, S. Psarras, T. Loutas, G. Sotiriadis, I. Gray, M.J. Padiyar, I. Petrunin, J. Raposo, L. ZanottiFragonara, V. Tzitzilonis, K. Dassios, D. Exarchos, G. Andrikopoulos, G. Nikolakopoulos, Autonomous Inspection and Repair of Aircraft Composite Structures, IFACPapersOnLine, Volume 51, Issue 30, 2018, Pages 554-557, ISSN 2405-8963.

https://doi.org/10.1016/j.ifacol.2018.11.267

[5] Hossein TOWSYFYAN, Ander BIGURI, Richard BOARDMAN, Thomas BLUMENSATH. Successes and challenges in non-destructive testing of aircraft composite structures. Chinese Journal of Aeronautics, Volume 33, Issue 3, 2020, Pages 771-791.

https://doi.org/10.1016/j.cja.2019.09.017

[6] S. Gholizadeh. A review of non-destructive testing methods of composite materials, Procedia Structural Integrity, 2016, vol. 1, pp. 50-57. https://doi.org/10.1016/j.prostr.2016.02.008 [7] Prakash, R.V., John, M., Post-impact Fatigue Damage Analysis of Quasi Isotropic CFRP Laminates through Infrared Thermography, Frattura ed Integrita Strutturale, 2019. https://doi.org/10.3221/IGF-ESIS.49.50

[8] Lison, M., Hendrick, P., Servais, P., Dufour, Y., Evaluation of NDT by robotic line scan thermography on composite air-craft parts, Structural Health Monitoring 2017: Real-Time Material State Awareness and Data-Driven Safety Assurance - Proceedings of the 11th International Workshop on Structural Health Monitoring, IWSHM 2017, 1, pp. 86-93. https://doi.org/10.12783/shm2017/13853

[9] Shakeb Deane, Nicolas P. Avdelidis, Clemente Ibarra-Castanedo, Hai Zhang, Hamed Yazdani Nezhad, Alex A. Williamson, Tim Mackley, Maxwell J. Davis, Xavier Maldague, Antonios Tsourdos, Application of NDT thermographic imaging of aerospace structures, Infrared Physics \& Technology, Volume 97, 2019, Pages 456-466.

https://doi.org/10.1016/j.infrared.2019.02.002

[10] James Moran, Nik Rajic. Remote line scan thermography for the rapid inspection of composite impact damage, Composite Structures, Volume 208, 2019, Pages 442-453. https://doi.org/10.1016/j.compstruct.2018.10.038 [11] FLIR Systems, Inc., "Why HOT MWIR Might Be a Better Fit than Uncooled LWIR", FLIR Systems, Inc., [Online]. Available: https://www.flir.com. [Accessed: Nov. 06, 2020]. 